\title{
Automatic Continuity in Associative and Nonassociative Context
}

\author{
A. R. VILLENA
}

\begin{abstract}
There are excellent accounts on automatic continuity theory in the associative context. Since there have been a number of advances in automatic continuity theory in the nonassociative context, it seems to be an opportune time to present a comprehensive discussion of the currently known results on automatic continuity of linear maps in this context. These notes are an attempt to collect together some of the results of automatic continuity theory (concentrating on homomorphisms and derivations) in associative and nonassociative context. Moreover, we present a number of open questions in both settings.
\end{abstract}

\section{InTRODUCTION}

In automatic continuity theory we are concerned with algebraic conditions on a linear map between Banach spaces which make this map automatically continuous. This theory has been mainly developed in the context of Banach algebras, and there are excellent accounts on automatic continuity theory $[16,17,58]$ (see also [42]) in this associative context. Since there have been a number of advances in the nonassociative context, it seems to be timely to compile a comprehensive list of currently known results on automatic continuity of linear maps in the nonassociative context. Research in automatic continuity mainly deals with two classes of linear maps on Banach algebras and nonassociative Banach algebras, which are well behaved with respect to the multiplicative structure: homomorphisms and

2000 Mathematics Subject Classification. 46-02, 46H40, 47B47, 17B40, $17 \mathrm{C} 65$.

Supported by D.G.I.C.Y.T. Grant PB98-1358.

The author would like to thank the organizers of the Belfast Functional Analysis Day 2000, Dr. Martin Mathieu and Prof. Anthony W. Wickstead for inviting him to deliver lectures on this paper. 
derivations. Jordan-Banach algebras and Lie-Banach algebras are the most important classes of nonassociative Banach algebras on which the automatic continuity of homomorphisms and derivations has been studied. These notes are an attempt to collect together the basic results. We emphasize that when considering the associative context, we are restricting our attention to those results that have (or are expected to have) a nonassociative extension. Moreover, we present a number of questions in both settings which seem to be open.

It should be pointed out that one of our main reasons for treating homomorphisms and derivations on nonassociative Banach algebras is that we believe this may prove to be useful for understanding homomorphisms and derivations on Banach algebras. Some results and especially some methods of the theory of noncommutative Banach algebras have proved to be useful in the study of commutative Banach algebras. Similarly, it may turn out that the study of homomorphisms and derivations on nonassociative Banach algebras will give a better picture on homomorphisms and derivations on noncommutative, associative Banach algebras, in particular on those questions in which the standard approaches have failed to give a solution.

\section{Preliminaries}

2.1. Nonassociative Banach Algebras. A nonassociative algebra is a linear space $A$ together with a bilinear map $(a, b) \mapsto a b$ from $A \times A$ into $A$, which we call the product of $A$. We use the term nonassociative algebra in order to emphasize that the associativity of the product of $A$ is not being assumed. We do not mean that associativity fails to hold, but only that associativity is not assumed to hold. The general references here are $[24,25,73]$. The most important classes of nonassociative algebras are associative algebras, Jordan algebras, and Lie algebras.

A Jordan algebra is a nonassociative algebra $J$ (over a field of characteristic different from 2) whose product $\cdot$ satisfies

$$
a \cdot b=b \cdot a \quad \text { and } \quad(a \cdot b) \cdot a^{2}=a \cdot\left(b \cdot a^{2}\right)
$$

for all $a, b \in J$. Jordan algebras were introduced in 1934 by P. Jordan, J. von Neumann, and E. Wigner motivated by quantum mechanics. Very important areas of applications of Jordan theory are 
Lie groups, foundations of geometry, and bounded symmetric domains in infinite dimensional holomorphy.

Every associative algebra $A$ becomes a Jordan algebra, denoted by $A^{+}$, with respect to the product $a \cdot b=\frac{1}{2}(a b+b a)$. A Jordan algebra is said to be special if it can be embedded into the derived Jordan algebra of an associative algebra, otherwise it is said to be exceptional. Besides $A^{+}$, the standard examples of Jordan algebras are $H(A, *)=\left\{a \in A: \quad a^{*}=a\right\}$ in the case when $A$ is endowed with a linear involution $*$ and the Jordan algebra $J(X, f)$ of a symmetric bilinear form $f$ on a linear space $X$ over a field $\mathbb{K}$. That is, $J(X, f)=$ $\mathbb{K} \oplus X$ endowed with the product given by $(\alpha+x) \cdot(\beta+y)=\alpha \beta+$ $f(x, y)+\beta x+\alpha y$ for all $\alpha, \beta \in \mathbb{K}$ and $x, y \in X$. It is important to know that there are examples of exceptional Jordan algebras such as the Albert algebra $H_{3}(\mathbb{O})$ which consists of all matrices of the form

$$
\left(\begin{array}{lll}
\lambda & x & y \\
\bar{x} & \mu & z \\
\bar{y} & \bar{z} & \nu
\end{array}\right)
$$

where $\lambda, \mu, \nu \in \mathbb{C}$ and $x, y, z \in \mathbb{O}$ (the complex octonions), with product given by $a \cdot b=\frac{1}{2}(a b+b a)$ for all $a, b \in H_{3}(\mathbb{O})$.

A Lie algebra is a nonassociative algebra $\mathfrak{L}$ whose product $[\cdot, \cdot]$ satisfies

$$
[a, a]=0 \quad \text { and } \quad[[a, b], c]+[[b, c], a]+[[c, a], b]=0
$$

for all $a, b, c \in \mathfrak{L}$. The main area of application of Lie algebras is Lie groups. One associates with every Lie group a Lie algebra in such a way that local problems concerning Lie groups are reduced to corresponding problems on Lie algebras.

Let $A$ be an associative algebra. Then $A$ becomes a Lie algebra, denoted by $A^{-}$, with respect to the product $[a, b]=a b-b a$. The standard examples of Lie algebras are the Lie subalgebras of an associative algebra such as $A$, the skew elements $K_{A}=\left\{a \in A: a^{*}=-a\right\}$ in the case when $A$ is endowed with a linear involution, and the derivation algebra $\mathfrak{D e r}(A)$ of $A$. In fact, the Poincaré-Birkhoff-Witt theorem states that every Lie algebra is a Lie subalgebra of an associative algebra.

A nonassociative Banach algebra is a nonassociative (real or complex) algebra $A$ whose underlying linear space is a Banach space with 
respect to a norm $\|\cdot\|$ satisfying

$$
\|a b\| \leq \gamma\|a\|\|b\|
$$

for all $a, b \in A$ and some $\gamma>0$. The most important classes of nonassociative Banach algebras are Banach algebras, Jordan-Banach algebras, and Lie-Banach algebras. As it is customary by a Banach algebra we mean an associative Banach algebra. By a Jordan-Banach algebra we mean a nonassociative Banach algebra whose underlying linear algebra is a Jordan algebra. By a Lie-Banach algebra we mean a nonassociative Banach algebra whose underlying algebra is a Lie algebra. For every Banach algebra $A, A^{+}$becomes a Jordan-Banach algebra and $A^{-}$becomes a Lie-Banach algebra (with $\gamma=2$ ). In the sequel we will consider complex algebras.

From now on, the left and right multiplication operators by $a \in A$ are the continuous linear operators $L_{a}, R_{a}: A \rightarrow A$ which are defined in the obvious way

$$
L_{a}(x)=a x \text { and } R_{a}(x)=x a
$$

for each $x \in A$. As it is usual, when $\mathfrak{L}$ is a Lie-Banach algebra, we write $\operatorname{ad}(a)(a \in \mathfrak{L})$ for the multiplication operator from $\mathfrak{L}$ into itself defined by $\operatorname{ad}(a)(x)=[a, x]$ for each $x \in \mathfrak{L}$.

2.2. Radicals. The basic automatic continuity problem for homomorphisms and derivations on Banach algebras often involves the notion of a radical. Therefore, we first review some of the standard facts on this concept.

Several different radicals are studied in algebra, but the Jacobson radical is definitely the most important in Banach algebra theory, and it is usually just called the radical. The $\operatorname{radical} \operatorname{Rad}(A)$ of an associative algebra $A$ is the largest ideal of $A$ consisting of quasiinvertible elements. We say that $A$ is semisimple if $\operatorname{Rad}(A)=0$ and we say that $A$ is radical if $\operatorname{Rad}(A)=A$.

The standard concept of invertibility in associative algebras was extended to the context of Jordan algebras by N. Jacobson. An element $a$ in a unital Jordan algebra $J$ is said to be invertible if there exists $b \in J$ such that $a \cdot b=1$ and $a^{2} \cdot b=a$. This is equivalent to the invertibility of the operator $U_{a}$ from $J$ to itself given by $U_{a}(x)=2 a \cdot(a \cdot x)-a^{2} \cdot x$ for each $x \in J$. The standard spectral theory can be extended to the context of Jordan-Banach 
algebras. This follows from the fact that if $J$ is a complex JordanBanach algebra and $a \in J$, then there exists a closed associative subalgebra $A$ of the unitisation $J^{1}$ of $J$ containing 1 and $a$. Therefore the spectral theory runs as in the associative case.

McCrimmon proved that in each Jordan algebra $J$ there exists a largest ideal consisting of quasi-invertible elements. This ideal is called the Jacobson-McCrimmon radical of $J$ and it will be denoted by $\operatorname{Rad}(J)$. We say that $J$ is semisimple if $\operatorname{Rad}(J)=0$. If $A$ is an associative algebra, then $\operatorname{Rad}\left(A^{+}\right)$coincides with the classical Jacobson radical of $A$.

In Lie context the radical may be defined as follows. If $I$ is an ideal of a Lie algebra $\mathfrak{L}$, then we define the derived sequence $I^{\prime}=[I, I]$ and $I^{(n+1)}=\left[I^{(n)}, I^{(n)}\right]$ for each $n \in \mathbb{N}$. The ideal $I$ is said to be solvable if there exists $n \in \mathbb{N}$ such that $I^{(n)}=0$. For every finite-dimensional Lie algebra $\mathfrak{L}$ there is a largest solvable ideal of $\mathfrak{L}$ which is called the radical of $\mathfrak{L}$. As far as we know there is no such device in the case of an infinite-dimensional Lie algebra $\mathfrak{L}$. It is natural to call a Lie algebra semisimple if it has no nonzero abelian ideal. Let us remark that the Lie-Banach algebra $A^{-}$is semisimple provided that $A$ is a semisimple Banach algebra whose centre equals zero. From now on $Z(A)$ denotes the centre of the associative algebra $A$. Of course, $Z(A) \subset \operatorname{Rad}\left(A^{-}\right)$for any reasonable notion of radical of a Lie algebra.

It should be noted that the (associative) strong radical makes sense for any nonassociative algebra. The strong radical of a nonassociative algebra, s-Rad $(A)$, of $A$ is the intersection of the maximal modular ideals of $A$, and $A$ is said to be strongly semisimple if $\mathrm{s}-\operatorname{Rad}(A)=0$. In the case where $A$ is either associative or Jordan, we have

$$
\operatorname{Rad}(A) \subset \operatorname{s-Rad}(A)
$$

\section{HOMOMORPHISMS}

Let $A$ and $B$ be nonassociative algebras. A homomorphism from $A$ into $B$ is a linear map $\Phi: A \rightarrow B$ with the property that

$$
\Phi(a b)=\Phi(a) \Phi(b)
$$

for all $a, b \in A$. In the case in which $A$ and $B$ are nonassociative Banach algebras the basic automatic continuity problem for homomorphisms is to impose algebraic conditions on the domain algebra 
$A$ or on the range algebra $B$ which make these maps automatically continuous.

A key notion for studying the continuity of a linear map $T$ from a Banach space $X$ into a Banach space $Y$ is that of the separating space $\mathfrak{S}(T)$ of $T$, which is defined as follows

$\mathfrak{S}(T)=\left\{y \in Y:\right.$ there exists $\left(x_{n}\right)$ in $X, x_{n} \rightarrow 0$ and $\left.T x_{n} \rightarrow y\right\}$.

$\mathfrak{S}(T)$ is a closed subspace of $Y$ that measures the closability of $T$. The closed graph theorem shows that $T$ is continuous if and only if $\mathfrak{S}(T)=0$. For a thorough discussion of the separating space we refer the reader to [58].

The use of the separating space in the study of homomorphisms between Banach algebras was developed by Rickart. It is worth pointing out that the separating space $\mathfrak{S}(\Phi)$ is a closed ideal of $B$ provided that $\Phi(A)$ is dense in $B$.

\subsection{Conditions on the range algebra in the associative con-}

text. The starting point for automatic continuity of homomorphisms is the continuity of every homomorphism $\Phi$ from any Banach algebra $A$ into $\mathbb{C}$. This property can be obviously derived from the fact that $\Phi(a) \in \operatorname{Sp}(a)$ for each $a \in A$. Here and subsequently, $\operatorname{Sp}(a)$ stands for the spectrum of $a$. This basic property immediately leads to the following result.

Theorem 3.1. (Gelfand [19], Silov [54]). Let $A$ be a Banach algebra, let $B$ be a semisimple commutative Banach algebra, and let $\Phi$ be a homomorphism from $A$ into $B$. Then $\Phi$ is continuous. Accordingly, every semisimple commutative Banach algebra carries a unique Banach algebra topology.

A simple example shows that the preceding theorem does not hold true when $B$ is not commutative, even if we replace semisimple by simple. Indeed, let $A$ be any infinite-dimensional Banach space made into a Banach algebra by defining all the products to be zero, let $f$ be a discontinuous linear functional on $A$, and let $\Phi$ be the map from $A$ into $M_{2}(\mathbb{C})$ defined by

$$
\Phi(a)=\left(\begin{array}{cc}
0 & f(a) \\
0 & 0
\end{array}\right)
$$

for each $a \in A$. Then $\Phi$ is a discontinuous homomorphism from $A$ into the simple Banach algebra $M_{2}(\mathbb{C})$. 
Theorem 3.2. (Rickart [44]). Let $A$ be a Banach algebra, let $B$ be a strongly semisimple Banach algebra, and let $\Phi$ be a homomorphism from $A$ onto a dense subalgebra of $B$. Then $\Phi$ is continuous. Accordingly, every strongly semisimple Banach algebra carries a unique Banach algebra topology.

This result is stronger than Theorem 3.1, but unfortunately this theorem is not strong enough to study the Banach algebra $L(X)$ for an infinite-dimensional Banach space $X$ because the strong radical of $L(X)$ contains the ideal $F(X)$ of operators with finite-dimensional range. Of course, the radical of $L(X)$ is zero.

Definitely, the most important result in this area is the famous theorem by Johnson.

Theorem 3.3. (Johnson [27]). Let $A$ be a Banach algebra, let $B$ be a semisimple Banach algebra, and let $\Phi$ be an epimorphism from $A$ onto B. Then $\Phi$ is continuous. Accordingly, every semisimple Banach algebra carries a unique Banach algebra topology.

Theorems 3.2 and 3.3 naturally lead to the following question.

Question 1. Let $A$ be a Banach algebra, let $B$ be a semisimple Banach algebra, and let $\Phi$ be a homomorphism from $A$ onto a dense subalgebra of $B$. Is $\Phi$ automatically continuous?

This problem is still open even in the case where $A$ and $B$ are $C^{*}$ algebras. It is easily seen that the preceding question is equivalent to the following one.

Question 2. Let $A$ and $B$ be Banach algebras and let $\Phi$ be a homomorphism from $A$ into $B$. Is $b$ quasinilpotent whenever $b \in \mathfrak{S}(\Phi)$ ?

In order to prove his theorem, Johnson used intensively irreducible representations. However, Aupetit brought spectral techniques into automatic continuity in [1], and he gave a beautiful proof of Johnson theorem based on the subharmonicity of the spectral radius in an essential way. His influential proof was followed up by Ransford in [43], who obtained a very simple and short proof of Johnson's theorem. This technique has proved to be very useful to study the automatic continuity of homomorphisms when dealing with conditions on the range algebra. The following result illustrates this technique; it can be easily derived from $[1,43]$ (see also [2]). 
Lemma 3.4. Let $X$ be a Banach space, let $B$ be a Banach algebra, and let $\Phi$ be a linear map from $X$ into $B$. Suppose that there exists $M>0$ such that $r(\Phi(x)) \leq M\|x\|$ for each $x \in X$. Then $r(\Phi(x))^{2} \leq$ $M\|x\| \operatorname{dist}(\Phi(x), \mathfrak{S}(\Phi))$ for each $x \in X$. Accordingly, $\mathfrak{S}(\Phi) \cap \Phi(X)$ consists of quasinilpotent elements.

In the preceding result and subsequently, $r(\cdot)$ stands for the spectral radius.

It is worth pointing out that the preceding result is the key fact to obtain nonassociative versions of Theorem 3.3. Let us illustrate this assertion with the following result.

Lemma 3.5. Let $A$ and $B$ be nonassociative Banach algebras and let $\Phi$ be an epimorphism from $A$ onto $B$. Then $\mathfrak{S}(\Phi)$ is a closed ideal of $B$, and the multiplication operators $L_{b}$ and $R_{b}$ on $B$ are quasinilpotent operators for each $b \in \mathfrak{S}(\Phi)$.

Proof. Let $\phi: A \rightarrow L(B)$ be the linear map defined by $\phi(a)=L_{\Phi(a)}$. For every $a \in A$, we have $\Phi \circ L_{a}=L_{\Phi(a)} \circ \Phi$. Since $\Phi$ is surjective, a standard result from spectral theory shows that $r\left(L_{\Phi(a)}\right) \leq r\left(L_{a}\right) \leq$ $\|a\|$. Consequently, $\phi$ satisfies the requirement in Lemma 3.4. Let $b \in \mathfrak{S}(\Phi)$. It is easily checked that $L_{b} \in \mathfrak{S}(\phi)$. On the other hand, there is $a \in A$ such that $\Phi(a)=b$ and therefore $\phi\left(L_{a}\right)=L_{b}$. From Lemma 3.4 it follows that $L_{b}$ is a quasinilpotent operator. In the same manner we can see that $R_{b}$ is a quasinilpotent operator.

\subsection{Conditions on the range algebra in the nonassociative}

context. First, let us remark that as far as we know it is not currently known whether or not Theorem 3.2 extends to general nonassociative Banach algebras.

Question 3. Let $A$ be a nonassociative Banach algebra, let $B$ be a strongly semisimple nonassociative Banach algebra, and let $\Phi$ be a homomorphism from $A$ onto a dense subalgebra of $B$. Is $\Phi$ automatically continuous?

It is easy to extend Theorem 3.2 (just the usual associative proof carries over) to Jordan-Banach algebras.

Theorem 3.6. Let $J$ be a Jordan-Banach algebra, let $H$ be a strongly semisimple Jordan-Banach algebra, and let $\Phi$ be a homomorphism from $J$ onto a dense subalgebra of $H$. Then $\Phi$ is continuous. 
Obviously, the canonical homomorphisms in Lie context are the so-called Lie homomorphisms between Banach algebras. If $A$ and $B$ are Banach algebras, then a homomorphism from the Lie algebra $A^{-}$into the Lie algebra $B^{-}$is said to be a Lie homomorphism from $A$ into $B$.

Surprisingly it seems to be not known whether or not Theorem 3.2 extends to the Lie context in the following sense.

Question 4. Let $A$ be a Banach algebra, let $B$ be a strongly semisimple Banach algebra, and let $\Phi$ be a Lie homomorphism from $A$ onto a dense subalgebra of $B$. Is $\mathfrak{S}(\Phi)$ contained in $Z(B)$ ?

Our next concern will be the nonassociative extensions of Johnson's theorem. Aupetit extended Theorem 3.3 to the Jordan context.

Theorem 3.7. (Aupetit [1]). Let $J$ be a Jordan-Banach algebra, let $H$ be a semisimple Jordan-Banach algebra, and let $\Phi$ be an epimorphism from $J$ onto $H$. Then $\Phi$ is continuous. Accordingly, every semisimple Jordan-Banach algebra carries a unique Jordan-Banach algebra topology.

Proof. Let $b \in \mathfrak{S}(\Phi)$. From Lemma 3.5 we have $r\left(L_{b}\right)=0$. On the other hand, we have

$$
\begin{aligned}
r(b) & =\lim \left\|b^{n+1}\right\|^{\frac{1}{n+1}}=\lim \left\|L_{b}^{n}(b)\right\|^{\frac{1}{n+1}} \\
& \leq \lim \left\|L_{b}^{n}\right\|^{\frac{1}{n+1}}\|b\|^{\frac{1}{n+1}}=r\left(L_{b}\right)
\end{aligned}
$$

and so $r(b)=0$. Therefore $\mathfrak{S}(\Phi)$ is an ideal of $B$ consisting of quasinilpotent elements, which gives $\mathfrak{S}(\Phi) \subset \operatorname{Rad}(H)=0$.

Since $\operatorname{Rad}\left(A^{+}\right)=\operatorname{Rad}(A)$ whenever $A$ is a Banach algebra, it follows that Theorem 3.3 can obviously be derived from the preceding theorem.

Theorems 3.3 and 3.7 were extended in [46] by observing that Aupetit's technique still works for the spectral subalgebras of a Banach algebra in the sense of [42]. This idea was used to canonically associate with any nonassociative algebra the so-called ultra-weak radical uw- $\operatorname{Rad}(A)$ and to prove the following.

Theorem 3.8. (Rodriguez [46]) Let A be a nonassociative Banach algebra, let $B$ be a nonassociative Banach algebra whose ultra-weak radical equals zero, and let $\Phi$ be an epimorphism from $A$ onto $B$. Then $\Phi$ is continuous. 
It is known that uw- $\operatorname{Rad}(A) \subset \operatorname{Rad}(A)$ in the case where $A$ is either a Banach algebra or a Jordan-Banach algebra. However, this theorem has not proved to be useful when dealing with Lie algebras, even with the most elementary examples of Lie algebras such as $A^{-}$ and $K_{A}$ for an associative algebra $A$.

The extension of Theorem 3.3 to the Lie context started by investigating the continuity of Lie isomorphisms between Banach algebras.

Theorem 3.9. (Berenguer-Villena [9]). Let $A$ and $B$ be semisimple Banach algebras, and let $\Phi$ be a Lie isomorphism from $A$ onto $B$. Then $\mathfrak{S}(\Phi)$ is contained in the centre of $B$.

The proof of the preceding theorem relied strongly on the structure of Lie isomorphisms and it was extended in [2] by removing the previously mentioned device. Here and subsequently, $\mathfrak{Z}(A)$ stands for the centre modulo the radical of a Banach algebra $A$, that is

$$
\mathfrak{Z}(A)=\{a \in A: \quad[a, A] \subset \operatorname{Rad}(A)\} .
$$

Theorem 3.10. (Aupetit-Mathieu [2]). Let $A$ and $B$ be Banach algebras, and let $\Phi$ be a Lie epimorphism from $A$ onto $B$. Then $\mathfrak{S}(\Phi) \subset \mathfrak{Z}(B)$. Accordingly, $\Phi$ is continuous provided that $B$ is semisimple and its centre is zero.

In fact, the same conclusion can be drawn when $A$ is a Lie-Banach algebra.

Proof. Let $b \in \mathfrak{S}(\Phi)$. Since $\mathfrak{S}(\Phi)$ is an ideal of $B^{-}$it follows that $[b, c] \in \mathfrak{S}(\Phi)$ and Lemma 3.5 now shows that ad $([b, c])$ is a quasinilpotent operator for each $c \in B$. The result follows from the following spectral characterization of the centre modulo the radical.

Lemma 3.11. ([2]). Let $A$ be a Banach algebra and let $a \in A$. Then $a \in \mathfrak{Z}(A)$ if and only if the operator $\operatorname{ad}([a, b])$ on $A$ is quasinilpotent for each $b \in A$.

This characterization has proved to be very useful even when dealing with the structure of derivation algebras on a Banach algebra [68].

It should be pointed out that Theorem 3.3 can be obtained as a consequence of Theorem 3.10 in the following way. Let $\Phi$ be an epimorphism from a Banach algebra $A$ onto a Banach algebra $B$. Then $\Phi$ extends to an epimorphism $\Phi_{1}$ from the unitisation $A^{1}=$ $A \oplus \mathbb{C}$ of $A$ onto the unitisation $B^{1}=B \oplus \mathbb{C}$ of $B$ and $\Phi_{1}$ lifts to an 
epimorphism $\Phi_{2}$ from the matrix algebra $M_{2}\left(A^{1}\right)$ onto the matrix algebra $M_{2}\left(B^{1}\right)$. On account of Theorem 3.10, $\left[\mathfrak{S}\left(\Phi_{2}\right), M_{2}\left(B^{1}\right)\right] \subset$ $\operatorname{Rad}\left(M_{2}\left(B^{1}\right)\right)$. For every $b \in \mathfrak{S}(\Phi)$, it is easily seen that $\left(\begin{array}{ll}b & 0 \\ 0 & 0\end{array}\right) \in$ $\mathfrak{S}\left(\Phi_{2}\right)$. Therefore $\left(\begin{array}{ll}0 & b \\ 0 & 0\end{array}\right)=\left[\left(\begin{array}{ll}b & 0 \\ 0 & 0\end{array}\right),\left(\begin{array}{ll}0 & 1 \\ 0 & 0\end{array}\right)\right] \in \operatorname{Rad}\left(M_{2}\left(B^{1}\right)\right)$. On the other hand, $\operatorname{Rad}\left(M_{2}\left(B^{1}\right)\right)=M_{2}\left(\operatorname{Rad}\left(B^{1}\right)\right)$ and so $b \in \operatorname{Rad}\left(B^{1}\right) \cap$ $B=\operatorname{Rad}(B)$ as required.

A second step in the study of automatic continuity of homomorphisms in Lie context was the investigation of the continuity of isomorphisms between the skew elements of some semisimple Banach algebras with involution.

Theorem 3.12. (Berenguer-Villena [8]). Let $A$ and $B$ be centrally closed prime Banach algebras with linear involution and assume that $B$ is in addition semisimple. If $\Phi$ is a Lie isomorphism from $K_{A}$ onto $K_{B}$, then $\Phi$ is continuous.

In order to prove the preceding result, we used methods from the structure theory of Lie maps, such as the fact that $\Phi$ extends to an (associative) isomorphism from the subalgebra $\left\langle K_{A}\right\rangle$ of $A$ generated by $K_{A}$ onto $\left\langle K_{B}\right\rangle$, and spectral methods in the spirit of [43].

We recall that a prime algebra $A$ is centrally closed if every linear map $f$ from a two-sided ideal $I$ of $A$ to $A$ with the property that $f(a b)=a f(b)$ and $f(b a)=f(b) a$ for all $a \in A$ and $b \in I$ is a multiple of the identity operator. It is known that primitive Banach algebras and prime $C^{*}$-algebras are centrally closed.

Of course, it is to be expected that a similar result holds true for epimorphisms from a Lie-Banach algebra onto the skew elements of a Banach algebra endowed with a linear involution.

Question 5. Let $\mathfrak{L}$ be a Lie-Banach algebra, let $A$ be a Banach algebra endowed with a continuous linear involution, and let $\Phi$ be a homomorphism from $\mathfrak{L}$ onto $K_{A}$. Does $\left[\mathfrak{S}(\Phi), K_{A}\right] \subset \operatorname{Rad}(A)$ ?

Clearly, we only need to obtain an spectral characterization of the radical of $K_{A}$ similar to that given in Lemma 3.11.

Question 6. Let $A$ be a Banach algebra endowed with a continuous linear involution and let $a \in K_{A}$ such that the operator $\operatorname{ad}([a, b])$ on $K_{A}$ is quasinilpotent for each $b \in A$. Is $\left[a, K_{A}\right]$ contained in $\operatorname{Rad}(A)$ ?

It is also natural to ask the following question. 
Question 7. Let $A$ be a Lie-Banach algebra, let $B$ be a semisimple Lie-Banach algebra, and let $\Phi$ be an epimorphism from $A$ onto $B$. Is $\Phi$ automatically continuous?

3.3. Conditions on the domain algebra. A key idea to study the continuity of a homomorphism $\Phi$ from a Banach algebra $A$ into a Banach algebra $B$ by imposing algebraic restrictions on the domain algebra $A$ is the so-called continuity ideal $\mathcal{C}(\Phi)$ of $\Phi$, which is defined by

$$
\mathcal{C}(\Phi)=\{a \in A: \quad \Phi(a) \mathfrak{S}(\Phi)=\mathfrak{S}(\Phi) \Phi(a)=0\} .
$$

Furthermore, a main tool in this theory is the main boundedness theorem of Badé and Curtis [3]. These authors prove that every homomorphism from $C(K)$ is continuous on some dense subalgebra of $C(K)$. In fact, they describe in great detail the structure of such a homomorphism.

Theorem 3.13. (Badé-Curtis [3]) Let $K$ be a compact Hausdorff space, let $B$ be a Banach algebra, and let $\Phi$ be a homomorphism from $C(K)$ into $B$. Then the following assertions hold.

i. The hull $\{\omega \in K: \quad f(\omega)=0 \forall f \in \mathcal{C}(\Phi)\}$ of the continuity ideal $\mathcal{C}(\Phi)$ is a finite subset $\left\{\omega_{1}, \ldots, \omega_{n}\right\}$ of $K$.

ii. The restriction of $\Phi$ to the dense subalgebra of $C(K)$ given by

$$
\left\{f \in C(K): \quad f \text { is constant near each } \omega_{j}, j=1, \ldots, n\right\}
$$

is continuous and therefore it extends to a continuous homomorphism $\Psi$ from $C(K)$ into $B$.

iii. $\Psi(C(K))$ is closed in $B, \operatorname{Rad}(\overline{\Phi(C(K))})=\mathfrak{S}(\Phi)$, and

$$
\overline{\Phi(C(K))}=\operatorname{Rad}(\overline{\Phi(C(K))}) \oplus \Psi(C(K)) .
$$

iv. The difference $\Theta=\Phi-\Psi$ when restricted to

$$
I=\left\{f \in C(K): \quad f\left(\omega_{j}\right)=0 \text { for each } j=1, \ldots, n\right\}
$$

is a homomorphism onto a dense subalgebra of $\operatorname{Rad}(\overline{\Phi(C(K))})$.

v. There are linear maps $\Theta_{1}, \ldots, \Theta_{n}: C(K) \rightarrow B$ such that $\Theta_{1}+\ldots+\Theta_{n}=\Theta$, the restriction of $\Theta_{j}$ to $\left\{f: f\left(\omega_{j}\right)=0\right\}$ is a homomorphism for each $j=1, \ldots, n$, and $\operatorname{Rad}(\overline{\Phi(C(K))})=\overline{\Theta_{1}(C(K))} \oplus \cdots \oplus \overline{\Theta_{n}(C(K))}$.

Part of Theorem 3.13 still holds for noncommutative $C^{*}$-algebras. 
Theorem 3.14. (Sinclair [57]). Let $A$ be a unital $C^{*}$-algebra, let $B$ be a Banach algebra, and let $\Phi$ be a homomorphism from $A$ into $B$. Then the following assertions hold.

i. $\overline{\mathcal{C}(\Phi)}$ has finite codimension in $A$.

ii. There is a finite-dimensional subspace $F$ of $A$ such that $A=$ $\overline{\mathcal{C}(\Phi)} \oplus F, \mathcal{C}(\Phi) \oplus F$ is a dense subalgebra of $A$, and the restriction of $\Phi$ to $\mathcal{C}(\Phi) \oplus F$ is continuous and therefore it extends to a continuous homomorphism $\Psi$ from $A$ into $B$.

iii. $\Psi(A)$ is closed in $B, \operatorname{Rad}(\overline{\Phi(A)}) \subset \mathfrak{S}(\Phi)$, and $B=\Psi(A) \oplus$ $\mathfrak{S}(\Phi)$.

iv. The difference $\Theta=\Phi-\Psi$ when restricted to $\overline{\mathcal{C}(\Phi)}$ is a homomorphism onto a dense subalgebra of $\mathfrak{S}(\Phi)$.

As far as we know there is not any extension of Theorems 3.13 and 3.14 to nonassociative $C^{*}$-algebras. For an account of nonassociative $C^{*}$-algebras we refer the reader to [47]. We recall that a $J B^{*}$-algebra is a complex Jordan-Banach algebra $J$ equipped with a (conjugatelinear) involution $*$ such that $\left\|U_{a}\left(a^{*}\right)\right\|=\|a\|^{3}$ for each $a \in J$.

Question 8. Let $J$ be a $J B^{*}$-algebra, let $H$ be a Jordan-Banach algebra, and let $\Phi$ be a homomorphism from $J$ into $H$. Is there a dense subalgebra of $J$ on which $\Phi$ is continuous?

\section{Derivations}

Derivations are defined by the familiar Leibniz formula and they clearly have their origin in the concept of differentiation. In this section, we shall see that they arise in a number of situations. A derivation on a nonassociative algebra $A$ is a linear map $D: A \rightarrow A$ such that

$$
D(a b)=D(a) b+a D(b)
$$

for all $a, b \in A$. When $A$ is a nonassociative Banach algebra the basic automatic continuity problem for derivations is to give algebraic conditions on $A$ which make these maps automatically continuous. It is easily checked that $\mathfrak{S}(D)$ is a closed ideal of $A$.

The important point to note here is that the spectral techniques have not proved to be useful in the study of the continuity of derivations. When considering the automatic continuity of derivations the so-called gliding hump argument is intensively used. This principle lies at the heart of many theorems on the automatic continuity of homomorphisms and derivations and it was brought into automatic 
continuity by Johnson and Sinclair $[28,30]$. For a thorough discussion of this and other basic principles in automatic continuity we refer the reader to [17]. The following result illustrates this technique.

Lemma 4.1. Let $X$ and $Y$ be Banach spaces, and let $T: X \rightarrow Y$ be a linear map. Suppose that there exist continuous linear maps $R_{n}: X \rightarrow X$ and continuous linear maps $Q_{n}$ from $Y$ into Banach spaces $Y_{n}$ such that $Q_{n} T R_{1} \cdots R_{n}$ is continuous for each $n \in \mathbb{N}$. Then there exists $m \in \mathbb{N}$ such that $Q_{n} T R_{1} \cdots R_{m}$ is continuous for each $n \in \mathbb{N}$.

4.1. Continuity of derivations in the associative context. Let us remark that the Singer-Wermer conjecture has been one of the main reasons to study the continuity of derivations. We shall discuss this subject later.

In 1958 Kaplansky conjectured that every derivation on a $C^{*}$ algebra is continuous [31] and that every derivation on a semisimple Banach algebra is continuous [32]. Sakai confirmed Kaplansky's conjecture for $C^{*}$-algebras in [50], Youngson extended this result to $J B^{*}$-algebras in [71], and finally Barton and Friedman extended it to $J B^{*}$-triple systems in [6]. The second conjecture by Kaplansky was confirmed by Johnson and Sinclair in [30].

Theorem 4.2. (Johnson-Sinclair [30]) Let $A$ be a semisimple Banach algebra and let $D$ be a derivation on $A$. Then $D$ is continuous.

This theorem was generalized by Jewell and Sinclair.

Theorem 4.3. (Jewell-Sinclair [26]) Let A be a Banach algebra such that:

i. For each infinite-dimensional closed ideal $I$ of $A$, there is a sequence $\left(a_{n}\right)$ in $A$ such that $\overline{I a_{n+1} \cdots a_{1}}$ is strictly contained in $\overline{I a_{n} \cdots a_{1}}$.

ii. A contains no nonzero finite-dimensional nilpotent ideal.

Then every derivation on $A$ is continuous.

We include a proof of this result in order to illustrate the use of the gliding hump argument.

Proof. Suppose that $\operatorname{dim} \mathfrak{S}(D)=\infty$. Then there is a sequence $\left(a_{n}\right)$ in $A$ such that $\overline{\mathfrak{S}(D) a_{n+1} \cdots a_{1}}$ is strictly contained in $\overline{\mathfrak{S}(D) a_{n} \cdots a_{1}}$ 
for each $n \in \mathbb{N}$. Let $R_{n}: A \rightarrow A$ be defined by $R_{n}(a)=a a_{n}$ for each $a \in A$ and let $Q_{n}$ be the quotient map from $A$ onto $A / \overline{\mathfrak{S}(D) a_{n} \cdots a_{1}}$. Since $Q_{n} \Phi R_{1} \ldots R_{n}$ is continuous for each $n \in \mathbb{N}$, there exists $n \in \mathbb{N}$ such that $Q_{n+1} \Phi R_{1} \ldots R_{n}$ is continuous. From this we deduce that $\mathfrak{S}(D) a_{n} \ldots a_{1} \subset \overline{\mathfrak{S}(D) a_{n+1} \cdots a_{1}}$, a contradiction. As $\operatorname{dim} \mathfrak{S}(D)<$ $\infty$, for every $b \in \mathfrak{S}(D)$ the map $a \mapsto D(a b)$ is continuous and therefore $\mathfrak{S}(D) b=0$. Therefore $\mathfrak{S}(D)^{2}=0$, which gives $\mathfrak{S}(D)=0$.

Jewell and Sinclair showed that every semisimple Banach algebra satisfies the conditions of the preceding theorem. Moreover this result applies to several interesting non-semisimple Banach algebras including the Volterra algebra $L_{1}(0,1)$, the weighted convolution algebra $L_{1}(\omega)$ for any weight function $\omega$ on $\mathbb{R}^{+}$, and Banach algebras of power series.

The main questions in the non-semisimple context are the following.

Question $9 . \quad$ i. Is every derivation on a semiprime Banach algebra automatically continuous?

ii. Is every derivation on a prime Banach algebra automatically continuous?

iii. Is the separating space of a derivation on a Banach algebra necessarily nilpotent?

Runde showed in [48] that all the preceding questions are equivalent in the commutative case. Mathieu and Runde observed in [40] that the same arguments also works in the noncommutative case and therefore that all the preceding questions are equivalent. Moreover Cusack showed in [15] that if the answer to any of the preceding questions is positive then every derivation on a Banach algebra leaves each primitive ideal invariant and otherwise, there exists a topologically simple radical Banach algebra. The question whether or not there exists such an algebra is one of the big open problems in Banach algebra theory. It should be pointed out that the preceding problems are still open even in the commutative case and that a commutative topologically simple radical Banach algebra leads to an example of a quasinilpotent operator with no hyperinvariant closed subspace.

Partial answers to the preceding question often involve a condition like $\cap_{n=1}^{\infty}(\operatorname{Rad}(A))^{n}=0$ (see [65]) because in such a situation one can put into action the Mittag-Leffler Theorem (see [16]). 
Theorem 4.4. (Villena [65]) Let $A$ be a Banach algebra, and let D be a derivation on $A$. Then $D$ is continuous provided that one of the following assertions holds.

i. $A$ is semiprime and $\operatorname{dim}\left(\operatorname{Rad}(A) \cap \bigcap_{n=1}^{\infty} A^{n}\right)<\infty$.

ii. $A$ is prime and $\operatorname{dim} \bigcap_{n=1}^{\infty}[a A \cap \operatorname{Rad}(A)]^{n}<\infty$ for some $a \in A$ with $a^{2} \neq 0$.

iii. $A$ is an integral domain and $\operatorname{dim} \bigcap_{n=1}^{\infty}[a A \cap \operatorname{Rad}(A)]^{n}<\infty$ for some non-zero $a \in A$.

Let us point out that a nonassociative approach to this problem may prove to be useful. Let $D$ be a derivation on a prime Banach algebra $A$. Consider $B$, the Banach space of all sequences $\left(a_{n}\right)$ in $A$ with $\lim a_{n}=0$ equipped with the product given by $\left(a_{n}\right)\left(b_{n}\right)=\left(0, a_{1} b_{1}, \ldots, a_{n} b_{n}, \ldots\right)$. We can define $d: B_{0} \rightarrow B$ by $d\left(a_{n}\right)=\left(D\left(a_{n}\right)\right)$, where $B_{0}=\left\{\left(a_{n}\right): a_{n}=0\right.$ for sufficiently large $\left.\mathrm{n}\right\}$. It is easily checked that $B$ is a prime nonassociative Banach algebra, that $B_{0}$ is a dense ideal of $B$, that $\bigcap_{n=1}^{\infty} B^{n}=0$, that $d$ is a derivation, and that $D$ is continuous just in the case when $d$ is closable. The difficulties with this approach are that $d$ is a partially defined derivation on $B$ and that associativity fails to hold for $B$.

Question 10. Let $A$ be a prime nonassociative Banach algebra with the property that $\bigcap_{n=1}^{\infty} A^{n}=0$, let $B$ be a dense ideal of $A$, and let $D: B \rightarrow A$ a derivation. Is $D$ closable?

The answer to this question is affirmative when $A$ is associative [65] and it is to be expected to be also true in the Jordan context.

We recall that partially defined derivations often arise in the $C^{*}$ algebraic formulation of quantum mechanics $([10,51])$. The time evolution of a system is modeled by a strongly continuous one parameter group of $*$-automorphisms whose generator is a derivation partially defined on the ambient $C^{*}$-algebra. In the case where we consider a one parameter group of isometries instead of $*$-automorphisms, the generator of the action is a derivation partially defined on the $J B^{*}$-triple system associated to the $C^{*}$-algebra. On the other hand, the automatic closability of the essentially defined derivations on semisimple Banach algebras [63] has proved to be useful to study the automatic continuity of the (everywhere) defined derivations on the Lie-Banach algebra of the skew elements of a Banach algebra with involution (Theorem 4.10). 
The continuity of derivations from a Banach algebra into a Banach bimodule arises in a number of situations. In particular it arises in cohomology theory of Banach algebras and also in the theory of extensions of Banach algebras [4]. Let $A$ be a Banach algebra. There are many classical theorems asserting that all derivations from $A$ into all, or some, Banach $A$-bimodules are automatically continuous under a variety of conditions on $A$. The fact that every derivation from a $C^{*}$-algebra into a Banach bimodule is continuous was proved by Ringrose in [45].

Theorem 4.5. (Ringrose [45]). Let $A$ be a $C^{*}$-algebra, let $X$ be a Banach $A$-bimodule, and let $D$ be a derivation from $A$ into $X$. Then $D$ is continuous.

4.2. Continuity of derivations in the nonassociative context. Sinclair proved in [56] that every continuous Jordan derivation on a semisimple Banach algebra is a derivation and he asked whether or not every Jordan derivation on a semisimple Banach algebra is continuous. ${ }^{1}$ Based on this question Aupetit [1] asked whether or not every derivation on a semisimple Jordan-Banach algebra is continuous. This question was solved in [62].

Theorem 4.6. (Villena [62]). Let $A$ be a semisimple Jordan-Banach algebra, and let $D$ be a derivation on $A$. Then $D$ is continuous.

Of course, Theorem 4.2 follows from the preceding theorem because $\operatorname{Rad}\left(A^{+}\right)=\operatorname{Rad}(A)$.

The proof of this result requires the powerful Zel'manov methods on Jordan algebras [72], such as the so-called imbedded pentad eaters (a very sophisticated algebraic device). Sometimes the problem can be associativized but in such a case we have to deal with noncomplete (associative) algebras. Let us point out that some of the devices introduced when dealing with this problem have proved to be useful to study the closability of partially defined derivations on Banach algebras. As a matter of fact, the author obtained the following result.

Theorem 4.7. (Villena [65]). Let $A$ be a Banach algebra, let $B$ be a subalgebra of $A$, and let $D$ be a derivation from $B$ into $A$. Suppose that $B A B \subset B$ and that $\operatorname{dim}\left(\operatorname{Rad}(A) \cap \bigcap_{n=1}^{\infty} B^{n}\right)<\infty$. Then $B(\mathfrak{S}(D) \cap B) B \subset \operatorname{Rad}(A)$.

\footnotetext{
${ }^{1}$ Editorial Note. Cusack showed in [14] that every Jordan derivation on a semiprime Banach algebra is in fact a derivation.
} 
The general problem of automatic continuity of derivations in the Lie context is the following.

Question 11. Let $\mathfrak{L}$ be a semisimple Lie-Banach algebra. Is every derivation on $\mathfrak{L}$ automatically continuous?

This question is still open. However, the automatic continuity of derivations in Lie context has been studied for some of the standard examples of Lie-Banach algebras such as $A^{-}$and $K_{A}$, where $A$ is a Banach algebra. The most elementary derivations in Lie context are the so-called Lie derivations, which are just the derivations of the Lie algebra $A^{-}$associated with an associative algebra $A$.

De la Harpe gave a very partial answer to the above-stated question by proving that every derivation on a classical Lie-Banach algebra of operators on a Hilbert space is continuous.

Theorem 4.8. (de la Harpe [20]). Let $\mathfrak{L}$ be a classical Lie-Banach algebra of operators on a Hilbert space and let $D$ be a derivation on $\mathfrak{L}$. Then $D$ is continuous.

Theorem 4.2 and part of Theorem 4.8 were extended to Lie derivations on Banach algebras in [7]. The remaining part of Theorem 4.8 was extended in [8] to a much more general context.

Theorem 4.9. (Berenguer-Villena [7]). Let $A$ be a semisimple $B a$ nach algebra, and let $D$ be a Lie derivation on $A$. Then $\mathfrak{S}(D)$ is contained in the centre of $A$.

The proof of the preceding result involves the gliding hump argument (Lemma 4.1) and modifications of methods used in the structure theory of Lie maps, such as in [11].

Let us point out that we can proceed similarly to the observation to Theorem 3.10 in order to see that Theorem 4.2 can be deduced from Theorem 4.9. Indeed, let $D$ be a derivation on a semisimple Banach algebra $A$. Then $D$ extends to a derivation $D_{1}$ on $A^{1}$ and this latter derivation lifts to a derivation $D_{2}$ on $M_{2}\left(A^{1}\right)$. On account of the above theorem, $\mathfrak{S}\left(D_{2}\right) \subset Z\left(M_{2}\left(A^{1}\right)\right)$. If $a \in \mathfrak{S}(D)$, then $\left(\begin{array}{ll}a & 0 \\ 0 & 0\end{array}\right) \in \mathfrak{S}\left(D_{2}\right)$ and therefore $\left(\begin{array}{ll}0 & a \\ 0 & 0\end{array}\right)=\left[\left(\begin{array}{ll}a & 0 \\ 0 & 0\end{array}\right),\left(\begin{array}{ll}0 & 1 \\ 0 & 0\end{array}\right)\right]=\left(\begin{array}{ll}0 & 0 \\ 0 & 0\end{array}\right)$, which gives $a=0$ as required.

Theorem 4.10. (Berenguer-Villena [8]). Let $A$ be a centrally closed prime semisimple Banach algebra with linear involution, and let $D$ be a derivation on $K_{A}$. Then $D$ is continuous. 
A question still unanswered is whether or not some of the requirements in the preceding theorem can be removed.

Question 12. Let $A$ be a semisimple Banach algebra with linear involution and let $D$ be a derivation on $K_{A}$. Does $\left[\mathfrak{S}(D), K_{A}\right]=0$ hold?

On the other hand, it should be pointed out that a semisimple Banach algebra may have discontinuous Lie derivations. Even in the Banach algebra $L(X)$ there are discontinuous Lie derivations for some Banach spaces $X$. In fact if $X$ is the Banach space constructed in [18], then every derivation from $L(X)$ into each Banach $L(X)$ bimodule is continuous and surprisingly there is a discontinuous Lie derivation on $L(X)$. The author has the conjecture that every Lie derivation on $L(X)$ is continuous provided that $X$ is a Banach space such that $X \cong X \oplus X$.

Question 13. Let $X$ be a Banach space such that $X \cong X \oplus X$. Is every Lie derivation on $L(X)$ automatically continuous?

As an attempt to extend Ringrose's theorem to the Jordan context, Hejazian and Niknam studied in [21] the continuity of derivations from a $J B^{*}$-algebra into a Jordan-Banach module. They found out that unfortunately there exist discontinuous derivations from some spin factors $J$ into some Jordan-Banach $J$-modules.

It is important to note that the continuity of nonassociative derivations from a Banach algebra into a Banach bimodule is often required in order to determine its structure. As an example, we present the following theorem by Johnson.

Theorem 4.11. (Johnson [29]). Let $A$ be a $C^{*}$-algebra, and let $X$ be a Banach A-bimodule. Then every continuous Jordan derivation from $A$ into $X$ is an associative derivation and every continuous Lie derivation from $A$ into $X$ is of the form $d+\tau$, where $d$ is an associative derivation from $A$ into $X$ and $\tau$ is a linear map from $A$ into $Z(X)$.

On account of Theorem 4.5 and the above theorem, it is natural to ask the following questions.

Question 14. Let $A$ be a $C^{*}$-algebra and let $X$ be a Banach $A$ bimodule.

i. Is every Jordan derivation from $A$ into $X$ continuous? 
ii. Is the separating space of every Lie derivation from $A$ into $X$ contained in the centre of $X$ ?

4.3. The Singer-Wermer conjecture in the associative context. A comprehensive account on this topic is given in [36].

Silov showed in [53] that $C^{\infty}([0,1])$ cannot be given a norm which makes it a Banach algebra. Kaplansky conjectured that the reason for this is that a commutative semisimple Banach algebra has no non-zero derivations. Singer and Wermer showed in [59] that every continuous derivation on a commutative Banach algebra maps into the radical and these authors conjectured that the condition of continuity was unnecessary. This question was subsequently called the Singer-Wermer conjecture.

Theorem 4.12. (Singer-Wermer [59]). Let $A$ be a commutative Banach algebra, and let $D$ be a continuous derivation on $A$. Then $D(A)$ is contained in the radical of $A$.

The Singer-Wermer conjecture has been one of the main motivations to investigate the automatic continuity of derivations on Banach algebras. As a matter of fact, Johnson studied the continuity of derivations on commutative Banach algebras to obtain the following result.

Theorem 4.13. (Johnson [28]). Let $A$ be a commutative Banach algebra with identity, and let $D$ be a derivation on $A$. Then there exist orthogonal idempotents $e_{0}, e_{1}, \ldots, e_{n}$ in $A$ such that $e_{0}+\cdots+$ $e_{n}=1$, such that $D\left(e_{0} A\right) \subset \operatorname{Rad}\left(e_{0} A\right)$, and such that each algebra $e_{1} A, \ldots, e_{n} A$ has just one maximal ideal. Moreover, if $A$ is semisimple, then $D$ is continuous.

From Johnson's theorem it is clear that in order to confirm the Singer-Wermer conjecture it suffices to consider derivations on the unitisation of a commutative radical Banach algebra. The SingerWermer conjecture was finally confirmed by Thomas.

Theorem 4.14. (Thomas [60]). Let $A$ be a commutative Banach algebra, and let $D$ be a derivation on $A$. Then $D(A)$ is contained in the radical of $A$.

There are some results which extend the Singer-Wermer theorem to noncommutative Banach algebras. One such generalization is the Kleinecke-Shirokov theorem. 
Theorem 4.15. (Kleinecke [33], Shirokov [52]) Let A be a Banach algebra, and let $a, b \in A$ such that $[a,[a, b]]=0$. Then $[a, b]$ is quasinilpotent.

The preceding result was independently obtained by Kleinecke and by Shirokov. It had been conjectured by Kaplansky on the base of a result by Jacobson [23] and some results by Wintner [70] and Wielandt [69], which were motivated by the question whether or not bounded observables can fulfill the Heisenberg uncertainty principle of quantum mechanics. Heisenberg's formalism identified the coordinates of particle momentum and position with operators $p_{i}$ and $q_{j}$ satisfying the canonical commutation relations

$$
p_{i} p_{j}-p_{j} p_{i}=q_{i} q_{j}-q_{j} q_{i}=0, \quad p_{i} q_{j}-q_{j} p_{i}=-i \hbar \delta_{i j} 1 .
$$

In the 1940s, it was of central interest as to whether or not the commutation relations could be realized by bounded linear operators on a Banach space $X$. Since every operator $a \in L(X)$ gives a derivation on the Banach algebra $L(X)$ defined by ad $(a)$, the question whether or not bounded observables can fulfill the Heisenberg uncertainty principle of quantum mechanics stimulated the study of derivations on Banach algebras. Wintner for the case of Hilbert spaces and Wielandt for general Banach spaces proved that this is impossible.

Note that we can express the bracket $[a,[a, b]]$ either in the way $-[a, \operatorname{ad}(b)(a)]$ or in the way $\operatorname{ad}(a)^{2}(b)$, and it turns out that we can replace either the inner derivation $x \mapsto[x, b]$ or the inner derivation $x \mapsto[a, x]$ in Theorem 4.15 by an arbitrary continuous derivation $D$ on $A$.

Theorem 4.16. (Mathieu-Murphy [39]). Let $A$ be a Banach algebra, and let $D$ be a continuous derivation on $A$. Then the following assertions hold.

i. $D(a)$ is quasinilpotent for every $a \in A$ such that $[D(a), a]=$ 0 .

ii. $D(b)$ is quasinilpotent for every $b \in A$ such that $D^{2}(b)=0$.

It is clear that the first assertion in the preceding theorem generalizes the Singer-Wermer theorem.

Another way to generalize the Singer-Wermer theorem to noncommutative Banach algebras was observed by Sinclair in [55]. This extension involves the notion of primitiveness. An ideal $P$ of an 
associative algebra $A$ is called primitive if it is the kernel of some irreducible representation of $A$ on some linear space $X$. If $A$ is not a radical algebra, then it is well known that $\operatorname{Rad}(A)$ is the intersection of all primitive ideals of $A$.

Theorem 4.17. (Sinclair [55]). Let $A$ be a Banach algebra, and let $D$ be a continuous derivation on $A$. Then $D$ leaves each primitive ideal of $A$ invariant.

It should be pointed out that Theorem 4.17 can be proved in much the same way as Theorems 4.15 and 4.16.

A number of authors have considered other properties of derivations on Banach algebras and extended the Singer-Wermer theorem in various directions. The question whether the continuity condition can be removed from each of these results has been of permanent interest and it has been a motivation for steady development of automatic continuity theory.

Thomas removed the continuity condition from Theorem 4.16.ii and he also proved that this property implies the truthfulness of the Singer-Wermer conjecture.

Theorem 4.18. (Thomas [61]). Let $A$ be a Banach algebra, and let $D$ be a derivation on $A$. Then $D(a)$ is quasinilpotent whenever $a \in A$ is such that $D^{2}(a)=0$.

However, it is still currently not known whether or not the continuity can be removed from Theorems 4.16.i and 4.17.

Question 15. Let $A$ be a Banach algebra, and let $D$ be a derivation on $A$. Is $D(a)$ quasinilpotent whenever $a \in A$ is such that $[D(a), a]=0$ ?

Question 16. Does every derivation on a Banach algebra leave each primitive ideal invariant?

The affirmative answer to question 15 is called ' the unbounded Kleinecke-Shirokov theorem'; it was first conjectured in [35]. One usually refers to the invariance of primitive ideals under derivations as to the noncommutative Singer-Wermer conjecture. It is worth pointing out that the truthfulness of the noncommutative Singer-Wermer conjecture implies the truthfulness of the unbounded Kleinecke-Shirokov theorem.

The following questions are clearly closely related to the noncommutative Singer-Wermer conjecture. 
Question 17. i. Does every derivation on a Banach algebra leave the radical invariant?

ii. Does every derivation on the unitisation of a radical Banach algebra leave the radical invariant?

We now consider a generalization of the unbounded KleineckeShirokov theorem and some questions closely related to it.

Question 18. Let $A$ be a Banach algebra, and let $D$ be a derivation on $A$. Is $D(a)$ quasinilpotent whenever $a \in A$ is such that $[D(a), a] \in$ $\operatorname{Rad}(A)$ ?

Question 19. Let $A$ be a Banach algebra and let $D$ be a derivation on $A$.

i. Does $[D(a), a] \in \operatorname{Rad}(A)$ for some $a \in A$ imply that $D(a)$ is quasinilpotent?

ii. Does $[D(a), a] \in \operatorname{Rad}(A)$ for each $a \in A$ imply that $D(A) \subset$ $\operatorname{Rad}(A)$ ?

iii. Does $[D(a), A] \subset \operatorname{Rad}(A)$ for some $a \in A$ imply that $D(a) \in$ $\operatorname{Rad}(A)$ ?

iv. Does $[D(A), A] \subset \operatorname{Rad}(A)$ imply that $D(A) \subset \operatorname{Rad}(A)$ ?

v. Does $[D(A), D(A)] \subset \operatorname{Rad}(A)$ imply that $D(A) \subset \operatorname{Rad}(A)$ ?

Surprisingly, Questions 16, 17, 18, and 19 are equivalent [13, 36]. Moreover Cusack [15] showed that if the answer to Question 16 is negative, then there exists a topologically simple radical Banach algebra. Let us remark that the answer to any of the preceding questions is affirmative for continuous derivations.

Thomas gave in [61] a partial answer to the noncommutative Singer-Wermer conjecture by studying which, and how many, primitive ideals can fail to be invariant.

Theorem 4.19. (Thomas [61]). Let $A$ be a Banach algebra, and let $D$ be a derivation on $A$. Then the following assertions hold

i. A primitive ideal $P$ of $A$ is invariant under $D$ if and only if $\mathfrak{S}\left(D^{n}\right) \subset P$ for each $n \in \mathbb{N}$.

ii. $D(P) \subset P$ for each primitive ideal $P$ of $A$ except possibly finitely many exceptional primitive ideals. Moreover, if $P$ is an exceptional primitive ideal then $P$ is finite-codimensional.

There are also some partial answers to the noncommutative SingerWermer conjecture in the commuting version. 
Theorem 4.20. (Mathieu [37]). Let $A$ be a Banach algebra, and let $D$ be a derivation on $A$ such that, for each $a \in A,[D(a), a] \in \operatorname{Nil}(A)$, the nil radical of $A$. Then $D(A) \subset \operatorname{Rad}(A)$.

There are also some partial versions of the unbounded KleineckeShirokov Theorem.

Theorem 4.21. (Runde [49]). Let $A$ be a Banach algebra, let $D$ be a derivation on $A$, and let $a \in A$ be such that $\left[a, D^{n}(a)\right]=0$ for each $n \in \mathbb{N}$. Then $D(a)$ is quasinilpotent.

In [12], a spectral approach to the noncommutative Singer-Wermer conjecture is proposed by proving the following result.

Theorem 4.22. (Brešar-Mathieu [12]). Let $A$ be a Banach algebra, and let $D$ be a derivation on $A$. Then $D(A) \subset \operatorname{Rad}(A)$ if and only if there is a constant $C$ such that $r(D(a)) \leq C r(a)$ for each $a \in A$.

We may summarize the above results to the statement that derivations on commutative Banach algebras are rather rare and that the same applies to derivations on general Banach algebras which satisfy certain commuting relations.

4.4. The Singer-Wermer conjecture in the nonassociative context. We now consider the Singer-Wermer conjecture in the Jordan context. In order to do this we first require the notion of primitive ideals in Jordan context.

It is known that an ideal $P$ of an associative algebra $A$ is primitive if there is a maximal modular left ideal $M$ of $A$ such that $P$ is the largest ideal of $A$ contained in $M$. In a similar way the primitive ideals of a Jordan algebra are defined. A linear subspace $I$ of a Jordan algebra $J$ is said to be an inner ideal of $J$ if $U_{I}\left(J^{1}\right) \subset I$. We say that an inner ideal $I$ in $J$ is $x$-modular for some $x \in J$ is $U_{1-x} J \subset I,\left\{1-x, J^{1}, I\right\} \subset I$, and $x-x^{2} \in I$, where $\{\cdot, \cdot, \cdot\}$ stands for the Jordan triple product $\{a, b, c\}=(a \cdot b) \cdot c+a \cdot(b \cdot c)-(a \cdot c) \cdot b$ for all $a, b, c \in J$. We call an ideal $P$ of $J$ primitive if it is the largest ideal of $J$ contained in a maximal modular inner ideal of $J$. It turns out that $\operatorname{Rad}(J)$ is the intersection of all primitive ideals of $J$ and that the primitive ideals of an associative algebra $A$ are primitive ideals of the Jordan algebra $A^{+}$. E. I. Zel'manov introduced the notion of primitiveness for unital Jordan algebras to derive his characterization of prime Jordan algebras. This concept was extended to nonunital Jordan algebras by L. Hogben and K. McCrimmon. 
Similarly to the associative context it is natural to ask the following question.

Question 20. Does every derivation on a Jordan-Banach algebra leave each primitive ideal invariant?

This question can be considered as the Singer-Wermer conjecture in Jordan context. Since every primitive ideal of a Banach algebra $A$ is a primitive ideal of $A^{+}$, it follows that the truthfulness of the Singer-Wermer conjecture in Jordan context implies the truthfulness of the noncommutative Singer-Wermer conjecture.

The following questions are clearly closely related to the SingerWermer conjecture in Jordan context as well as to Question 17.

Question 21. i. Does every derivation on a Jordan-Banach algebra leave the radical invariant?

ii. Does every derivation on the unitisation of a radical JordanBanach algebra leave the radical invariant?

On the other hand, similarly to the associative context we should expect that derivations of Jordan-Banach algebras can only exceptionally satisfy some associating relations. We set

$$
[a, b, c]=(a \cdot b) \cdot c-a \cdot(b \cdot c)
$$

for the associator of elements $a, b, c$ in a Jordan-Banach algebra $J$. Let us remark that Jordan associators are closely related to associative commutators. In the case where $J=A^{+}$we have

$$
[a, b, c]=\frac{1}{4}[[c, a], b] .
$$

Question 22. Let $J$ be a Banach algebra and let $D$ be a derivation on $J$.

i. Does $[D(a), J, a] \in \operatorname{Rad}(J)$ for some $a \in J$ imply that $D(a)$ is quasinilpotent?

ii. Does $[D(a), J, a] \in \operatorname{Rad}(J)$ for each $a \in J$ imply that $D(J) \subset$ $\operatorname{Rad}(J)$ ?

iii. Does $[D(a), J, J] \subset \operatorname{Rad}(J)$ for some $a \in J$ imply that $D(a)$ is quasinilpotent?

iv. Does $[D(J), J, J] \subset \operatorname{Rad}(J)$ imply that $D(J) \subset \operatorname{Rad}(A)$ ?

v. Does $[D(J), D(J), D(J)] \subset \operatorname{Rad}(J)$ imply that $D^{2}(A)$ consists of quasinilpotent elements? 
It is quite apparent that the preceding questions are closely related to the corresponding ones in Question 19. Brešar and the author recently proved in [13] that Questions 20, 21, and 22 are equivalent. It is also natural to ask the following.

Question 23. Suppose that the noncommutative Singer-Wermer conjecture is true and that the Singer-Wermer conjecture in Jordan context is false. What kind of pathological Jordan-Banach algebra arise in this situation?

A partial answer to the problem of invariance of primitive ideals was given in [62]. This result extends Theorem 4.19. A partial answer to the problem of associating derivations was given in [13].

Theorem 4.23. (Villena [62]) Let $J$ be a Jordan-Banach algebra, and let $D$ be a derivation on $J$. Then the following assertions hold.

i. A primitive ideal $P$ of $J$ is invariant under $D$ if and only if $\mathfrak{S}\left(D^{n}\right) \subset P$ for each $n \in \mathbb{N}$.

ii. $D(P) \subset P$ for each primitive ideal $P$ of $J$ except possibly finitely many exceptional primitive ideals. Moreover, if $P$ is an exceptional primitive ideal then $J / P$ is simple and it is either finite-dimensional or it is the Jordan-Banach algebra of a continuous nondegenerate symmetric bilinear form $f$ on a complex Banach space $X$ of dimension greater than one.

iii. If $D$ is continuous, then $D(P) \subset P$ for each primitive ideal $P$ of $J$.

Theorem 4.24. (Brešar-Villena [13]) Let $J$ be a complex JordanBanach algebra, and let $D$ be a derivation on $J$. Then the following assertions hold.

i. Suppose that $[D(a), J, a]=0$ for each $a \in J$. Then $D(J) \subset$ $\operatorname{Rad}(J)$.

ii. Suppose that $[D(a), J, a] \subset \operatorname{Rad}(J)$ for each $a \in J$. Then there are pairwise orthogonal idempotents $e_{1}, \ldots, e_{n}$ in $J$ such that

$$
D(J) \subset \mathbb{C} e_{1}+\cdots+\mathbb{C} e_{n}+\operatorname{Rad}(J) .
$$

iii. Suppose that $[D(J), D(J), D(J)]=0$. Then $D^{2}(J)$ consists of quasinilpotent elements.

iv. Suppose that $[D(J), D(J), D(J)] \subset \operatorname{Rad}(J)$. Then there are pairwise orthogonal idempotents $e_{1}, \ldots, e_{n}$ in $J$ such that

$$
D^{2}(J) \subset \mathbb{C} e_{1}+\cdots+\mathbb{C} e_{n}+\mathcal{Q}(J),
$$


where $\mathcal{Q}(J)$ denotes the set of all quasinilpotent elements of $J$.

Let us mention that there are non-zero derivations $D$ on simple Jordan-Banach algebras $J$ such that $D^{2}(a)=0=D(a)^{2}$ for all $a \in J$. This shows that a well-known result of Posner fails to be true in Jordan context and that there exist derivations on (even simple) Jordan-Banach algebras $J$ whose range consists of quasinilpotent elements but it does not lie in the radical. Hence Theorem 4.22 fails to be true in the Jordan context.

We leave as an open question whether the appearance of idempotents can be removed in Theorem 4.24. This question is equivalent to the Singer-Wermer conjecture in Jordan context.

A standard approach when treating a possibly discontinuous derivation $D$ is to consider its separating space and often it is also necessary to treat the separating spaces of its powers. Brešar and the author introduced in [13] the closed ideal $\mathfrak{I}(D)$ of $A$ generated by

$$
\left\{\mathfrak{S}\left(D^{n}\right): \quad n \in \mathbb{N}\right\}
$$

The reason to do this is that $\mathfrak{S}(D)$ is a closed ideal of $J$, but $\mathfrak{S}\left(D^{n}\right)$ does not have such nice algebraic structure in case $n>1$. This device has proved to be very useful when dealing with the range of a derivation. We shall illustrate how $\mathfrak{I}(D)$ works. As a matter of fact, if $[D(a), J, a]=0$ for each $a \in J$ then we can see that $[\Im(D), J, J]=0$. Moreover $[\Im(D), \Im(D), \Im(D)]=0$ provided that $[D(J), D(J), D(J)]=0$. In each case $\mathfrak{I}(D)$ is a commutative Banach algebra and for every $y \in \mathfrak{I}(D)$ the map

$$
d: \mathfrak{I}(D) \rightarrow \mathfrak{I}(D), \quad d(a)=D(a) \cdot y
$$

is a derivation of $\Im(D)$. Therefore we can apply Theorem 4.14 in order to obtain information about $D$.

It is important to realise that similar arguments apply to the case in which $D$ is a derivation on a Banach algebra that satisfies some commuting relation.

A possible approach to the noncommutative Singer-Wermer conjecture in the Lie context is by considering the structure of Lie derivations in the spirit of Herstein programme.

The simplest examples of derivations in the Lie context are Lie derivations on Banach algebras. It is clear that any associative derivation is a Lie derivation and the main interest in the study of 
the structure of Lie derivations is how similar are Lie derivations to associative derivations. This question was suggested by Herstein [22] in the frame of simple rings. There are some theorems $[5,11,34]$ which state that, if some requirements are satisfied, then any Lie derivation $D$ on a ring $R$ has the form $d+\tau$, where $d$ is an associative derivation from the ring $R$ to an enlargement $R^{\prime}$ of the ring and $\tau$ is an additive map from the ring to the center of the enlargement. The problem is that the enlargement may be too large. In fact this enlargement is usually too large to be useful in the analytical study of Lie derivations on Banach algebras. The most perfect decomposition of a Lie derivation would be a decomposition of the form $D=d+\tau$ in such a way that $d$ is an associative derivation from the ring to itself. We call such a Lie derivation a standard Lie derivation. There are some theorems $[29,38,41]$ which asserts that any Lie derivation on some special classes of Banach algebras are standard. From the work by Johnson we know that every continuous Lie derivation on a $C^{*}$-algebra is standard.

Theorem 4.25. (Johnson [29]) Let D be a continuous Lie derivation on a $C^{*}$-algebra $A$. Then $D$ is of the form $d+\tau$, where $d$ is a derivation on $A$ and $\tau$ is a linear map from $A$ into the centre of $A$.

It is important to know that unfortunately there are examples of discontinuous Lie derivations on some $C^{*}$-algebras. Indeed, if $A=\ell_{\infty}\left(M_{2}(\mathbb{C})\right)$ then $\operatorname{codim}[A, A]=\infty$. Therefore there exists a discontinuous linear functional $\tau$ on $A$ such that $\tau([A, A])=0$ which gives a discontinuous Lie derivation on $A$.

Consequently, one may ask whether Johnson's theorem is still true if we consider discontinuous derivations on $C^{*}$-algebras.

Question 24. Is every (possibly discontinuous) Lie derivation on a $C^{*}$-algebra standard?

It was proved in [66] that every Lie derivation on an arbitrary Banach algebra is standard modulo almost all the primitive ideals. We call a Lie derivation $D$ on a Banach algebra $A$ standard modulo a primitive ideal $P$ if there exist an associative derivation $d_{P}$ on $A / P$ and a linear map $\tau_{P}$ from $A$ into the centre of $A / P$ such that

$$
Q_{P} D(a)=d_{P}(a+P)+\tau_{P}(a) \quad(a \in A),
$$

where $Q_{P}$ stands for the quotient map from $A$ onto $A / P$. 
Theorem 4.26. (Villena [66]). Let $A$ be a unital complex Banach algebra, and let $D$ be a Lie derivation on $A$. Then the following assertions hold.

i. $D$ is standard modulo a primitive ideal $P$ if and only if $\left[\mathfrak{S}\left(D^{n}\right), A\right] \subset P$ for each $n \in \mathbb{N}$.

ii. $D$ is standard modulo $P$ for each primitive ideal $P$ except possibly finitely many exceptional primitive ideals. Moreover, if $P$ is an exceptional primitive ideal then $P$ has finite codimension greater than one.

iii. If $D$ is continuous, then $D$ is standard modulo $P$ for each primitive ideal $P$.

iv. $D$ is standard modulo a primitive ideal $P$ if and only if $D(P) \subset \mathbb{C}+P$.

The proof of the preceding result combines ideas in both [11] and [61].

One may ask whether there are actually no exceptional primitive ideals for the decomposition of Lie derivations given in the preceding theorem. We can state the following open question which can be considered as the Singer-Wermer conjecture in Lie context.

Question 25. Let $A$ be a unital complex Banach algebra and let $D$ be a Lie derivation on $A$. Is $D(P)$ contained in $\mathbb{C}+P$ for each primitive ideal $P$ of $A$ ?

We note that it is easy to show that the truthfulness of the SingerWermer conjecture in Lie context implies the truthfulness of the noncommutative Singer-Wermer conjecture. Indeed, assume that the answer to Question 25 is affirmative and let $D$ be a derivation on the unitisation $R^{1}$ of a radical Banach algebra $R$. Then $D$ lifts to a derivation $\Delta$ on $M_{2}\left(R^{1}\right)$. Since $M_{2}\left(R^{1}\right) / M_{2}(R) \cong M_{2}\left(R^{1} / R\right) \cong$ $M_{2}(\mathbb{C})$, it follows that $M_{2}(R)$ is a maximal two-sided ideal of $M_{2}\left(R^{1}\right)$ and so

$$
\left(\begin{array}{ll}
D(R) & D(R) \\
D(R) & D(R)
\end{array}\right)=\Delta\left(M_{2}(R)\right) \in \mathbb{C}\left(\begin{array}{ll}
1 & 0 \\
0 & 1
\end{array}\right)+\left(\begin{array}{ll}
R & R \\
R & R
\end{array}\right) .
$$

Hence $D(R) \subset R$. This gives an unexpected intimate connection between the structure of Lie derivations and the noncommutative Singer-Wermer conjecture. On the other hand, it is natural to ask the following. 
Question 26. Suppose that the noncommutative Singer-Wermer conjecture is true and that the Singer-Wermer conjecture for Lie derivations is false. What kind of pathological Banach algebra arises in this situation?

Let $A$ be a semisimple Banach algebra and let $D$ be a Lie derivation on $A$. It can be proved that $D$ is standard modulo all the primitive ideals and that $D$ is standard if and only if for every $a \in A$ there exists $\tau(a) \in A$ such that $Q_{P}(\tau(a))=\tau_{P}(a)$ for each primitive ideal $P$ of $A$. It should be pointed out that in the case in which $A$ is a $C^{*}$-algebra this latter condition is equivalent to the continuity of the map $P \mapsto \tau_{P}(a)$ from the set $\operatorname{Prim}(A)$ of all primitive ideals of $A$ (endowed with the Jacobson topology) into $\mathbb{C}$ for each $a \in A$.

\section{REFERENCES}

[1] B. Aupetit, The uniqueness of the complete norm topology in Banach algebras and Banach Jordan algebras. J. Funct. Anal. 47 (1982), 1-6.

[2] B. Aupetit and M. Mathieu, The continuity of Lie homomorphisms. Studia Math. 138 (2000), 193-199.

[3] W. G. Badé and P. C. Curtis, Homomorphisms of commutative Banach algebras. Amer. J. Math. 82 (1960), 589-608.

[4] W. G. Badé, H. G. Dales, and Z. A. Lykova, Algebraic and strong splittings of extensions of Banach algebras. Mem. Amer. Math. Soc. 137 (1999).

[5] R. Banning and M. Mathieu, Commutativity preserving mappings on semiprime rings. Commun. Algebra 25 (1997), 247-265.

[6] T. J. Barton and Y. Friedman, Bounded derivations of $J B^{*}$-triples. Quart. J. Math. Oxford 41 (1990), 255-268.

[7] M. I. Berenguer and A. R. Villena, Continuity of Lie derivations on Banach algebras. Proc. Edinburgh Math. Soc. 41 (1998), 625-630

[8] M. I. Berenguer and A. R. Villena, Continuity of Lie mappings of the skew elements of Banach algebras with involution. Proc. Amer. Math. Soc. 126 (1998), 2717-2720.

[9] M. I. Berenguer and A. R. Villena, Continuity of Lie isomorphisms of Banach algebras. Bull. London Math. Soc. 31 (1999), 6-10.

[10] O. Bratteli, Derivations, dissipations and group actions on $C^{*}$ algebras. Lecture Notes in Mathematics 1229, Springer-Verlag, 1986.

[11] M. Brešar, Commuting traces of biadditive mappings, commutativitypreserving mappings and Lie mappings. Trans. Amer. Math. Soc. 335 (1993), 525-546. 
[12] M. Brešar and M. Mathieu, Derivations mapping into the radical, III. J. Funct. Anal. 133 (1995), 21-29.

[13] M. Brešar and A. R. Villena, The range of a derivation on a JordanBanach algebra. Studia Math., to appear.

[14] J. M. Cusack, Jordan derivations on rings, Proc. Amer. Math. Soc. 53 (1975), 321-324.

[15] J. M. Cusack, Automatic continuity and topologically simple radical Banach algebras. J. London Math. Soc. 16 (1977), 493-500.

[16] H. G. Dales, Automatic continuity: a survey. Bull. London Math. Soc. 10 (1978), 129-183.

[17] H. G. Dales, Banach algebras and automatic continuity. London Mathematical Society Monographs, Oxford University Press, 2000.

[18] H. G. Dales, R. J. Loy, and G. A. Willis, Homomorphisms and derivations from $B(E)$. J. Funct. Anal. 120 (1994), 201-219.

[19] I. M. Gelfand, Normierte Ringe. Rec. Math. (Mat. Sbornik) 51 (1941), 3-24.

[20] P. de la Harpe, Classical Banach-Lie algebras and Banach Lie groups of operators in Hilbert space. Lect. Notes in Math. 285, SpringerVerlag, Berlin 1972.

[21] S. Hejazian and A. Niknam, Modules, annihilators and module derivations of JB*-algebras. Indian J. Pure Appl. Math. 27 (1996), 129-140.

[22] I. N. Herstein, Lie and Jordan structures in simple, associative rings. Bull. Amer. Math. Soc. 67 (1961), 517-531.

[23] N. Jacobson, Rational methods in the theory of Lie algebras. Ann. of Math. 36 (1935), 875-881.

[24] N. Jacobson, Lie algebras. Dover Publications Inc., New York 1962.

[25] N. Jacobson, Structure and representation of Jordan algebras. AMS Colloq. Publ. Vol. 39, Providence, 1968.

[26] N. P. Jewell and A. M. Sinclair, Epimorphisms and derivations on $L_{1}(0,1)$ are continuous. Bull. London Math. Soc. 8 (1976), 135-139.

[27] B. E. Johnson, The uniqueness of the (complete) norm topology. Bull. Amer. Math. Soc. 73 (1967), 537-539.

[28] B. E. Johnson, Continuity of derivations on commutative Banach algebras. Amer. J. Math. 91 (1969), 1-10.

[29] B. E. Johnson, Symmetric amenability and nonexistence of Lie and Jordan derivations. Math. Proc. Camb. Phil. Soc. 120 (1996), 455473.

[30] B. E. Johnson and A. M. Sinclair, Continuity of derivations and a problem of Kaplansky. Amer. J. Math. 90 (1968), 1067-1073.

[31] I. Kaplansky, Functional analysis. Some aspects of analysis and probability, 1-34, Surveys in Applied Mathematics. Vol. 4. John Wiley \& Sons, Inc., New York: Chapman \& Hall, Ltd, London, 1958. 
[32] I. Kaplansky, Derivations of Banach algebras. Seminar on Analytic Functions. Vol. II. Institute for Advanced Study, Princeton, 1958.

[33] D. C. Kleinecke, On operator commutators. Proc. Amer. Math. Soc. 8 (1957), 535-536.

[34] W. S. Martindale, Lie derivations of primitive rings. Michigan J. Math. 11 (1964), 183-187.

[35] M. Mathieu, Is there an unbounded Kleinecke-Shirokov theorem? Sem. ber. Funkt. anal. 18, Tübingen, 1990, 137-143.

[36] M. Mathieu, Where to find the image of a derivation. Banach Centre Publications 30, Warsaw 1994, 237-249.

[37] M. Mathieu, On the range of centralizing derivations. Second International Conference on Algebra (Barnaul, 1991), 291-297, Contemp. Math., 184, Amer. Math. Soc., Providence, RI, 1995.

[38] M. Mathieu, Lie mappings of $C^{*}$-algebras. In: Nonassociative algebra and its applications. R. Costa et al (eds.), Marcel Dekker, New York $2000,229-234$.

[39] M. Mathieu and G. J. Murphy, Derivations mapping into the radical. Arch. Math. (Basel) 57 (1991), 469-474.

[40] M. Mathieu and V. Runde, Derivations mapping into the radical, II. Bull. London Math. Soc. 24 (1992), 485-487.

[41] C. R. Miers, Lie derivations of von Neumann algebras. Duke Math. 40 (1973), 403-409.

[42] T. W. Palmer, Banach algebras and the general theory of $*$-algebras, Volume I, Algebras and Banach algebras. Cambridge University Press 1994.

[43] T. J. Ransford, A short proof of Johnson's uniqueness-of-norm theorem. Bull. London. Math. Soc. 21 (1989), 487-488.

[44] C. E. Rickart, The uniqueness-of-norm problem in Banach algebras. Ann. of Math. 51 (1950), 615-628.

[45] J. R. Ringrose, Automatic continuity of derivations of operator algebras. J. London Math. Soc. 5 (1972), 432-438.

[46] A. Rodríguez, The uniqueness of the complete norm topology in complete normed nonassociative algebras. J. Funct. Anal. 60 (1985), 1-15.

[47] A. Rodríguez, Jordan structures in analysis, in: Jordan Algebras, Proc. Conf. Oberwolfach, August 9-15, 1992, W. Kaup, K. McCrimmon, and H. Peterson (eds.), de Gruyter, Berlin, 1994, 97-186.

[48] V. Runde, Automatic continuity of derivations and epimorphisms. Pacific J. Math. 147 (1991), 365-374.

[49] V. Runde, Range inclusion results for derivations on noncommutative Banach algebras. Studia Math. 105 (1993), 159-172.

[50] S. Sakai, On a conjecture of Kaplansky. Tôhoku Math. J. 12 (1960), 31-33. 
[51] S. Sakai, Operator algebras in dynamical systems. Cambridge University Press, 1991.

[52] F. V. Shirokov, Proof of a conjecture of Kaplansky. Uspekhi Mat. Nauk. 11 (1956), 167-168.

[53] G. E. Silov, On a property of rings of functions. Dokl. Akad. Nauk. SSSR, 58 (1947), 985-988.

[54] G. E. Silov, On regular normed rings. Trav. Inst. Math. Stekloff 21, 1947.

[55] A. M. Sinclair, Continuous derivations on Banach algebras. Proc. Amer. Math. Soc. 20 (1969), 166-170.

[56] A. M. Sinclair, Jordan homomorphisms and derivations on semisimple Banach algebras. Proc. Amer. Math. Soc. 24 (1970), 209-214.

[57] A. M. Sinclair, Homomorphisms from $C^{*}$-algebras. Proc. London Math. Soc. 29 (1974), 435-452; Corrigendum 32 (1976), 322.

[58] A. M. Sinclair, Automatic continuity of linear operators. Cambridge University Press 1976.

[59] I. M. Singer and J. Wermer, Derivations on commutative normed algebras. Math. Ann. 129 (1955), 260-264.

[60] M. P. Thomas, The image of a derivation is contained in the radical. Ann. of Math. 128 (1988), 435-460.

[61] M. P. Thomas, Primitive ideals and derivations on non-commutative Banach algebras. Pacific J. Math. 159 (1993), 139-152.

[62] A. R. Villena, Derivations on Jordan-Banach algebra. Studia Math. 118 (1996), 205-229.

[63] A. R. Villena, Essentially defined derivations on semisimple Banach algebras. Proc. Edinburgh Math. Soc. 40 (1997), 175-179.

[64] A. R. Villena, Derivations with a hereditary domain. J. London Math. Soc. 57 (1998), 469-477.

[65] A. R. Villena, Derivations with a hereditary domain, II. Studia Math. 130 (1998), 275-291.

[66] A. R. Villena, Lie derivations on Banach algebras. J. Algebra 226 (2000), 390-409.

[67] A. R. Villena, A local property of derivations on Jordan-Banach algebras. Commun. Algebra, to appear.

[68] A. R. Villena, Epimorphisms onto derivation algebras. Preprint.

[69] H. Wielandt, Über die Unbeschränktheit der Operatoren der Quantenmechanik. Math. Ann. 121 (1949/50), 21.

[70] A. Wintner, The unboundedness of quantum-mechanical matrices. Phys. Rev. 71 (1947), 738-739.

[71] M. A. Youngson, Hermitian operators on Banach Jordan algebras. Proc. Edinburgh Math. Soc. 22 (1979), 93-104.

[72] E. I. Zel'manov, On prime Jordan algebras II. Siberian Math. J. 24 (1983), 89-104. 
[73] K. A. Zhevlakov, A. M. Slinko, I. P. Shestakov, and A. I. Shirshov, Rings that are nearly associative. Academic Press, 1982.

\author{
A. R. Villena, \\ Departamento de Análisis Matemático, \\ Facultad de Ciencias, \\ Universidad de Granada, \\ E-18071 Granada, Spain, \\ avillena@ugr.es
}

Received on 30 May 2001. 